\title{
The Effects of Food-Related Retail Ambient Scents on Healthy/Unhealthy Food Purchases: An Abstract
}

\author{
Dipayan Biswas and Courtney Szocs
}

\begin{abstract}
Retailers use ambient scent as a strategic tool to create an overall ambience and influence consumers. Food-related ambient scents are especially common in stores and restaurants. This research examines how indulgent (i.e., unhealthy) as opposed to non-indulgent (i.e., healthy) food-related ambient scents influence consumers' food choices. The results of three field studies with children and adult consumers show that indulgent (vs. non-indulgent) food-related ambient scents lead to lower sales of unhealthy food items. Follow-up studies replicate this finding in the laboratory and provide evidence for cross-modal sensory compensation as the process driving the effects of indulgent food-related ambient scents on unhealthy food choices. Implications for retail design and consumer well-being are discussed.
\end{abstract}

\author{
D. Biswas \\ University of South Florida, Tampa, FL, USA \\ e-mail: dbiswas@usf.edu \\ C. Szocs $(\bowtie)$ \\ Portland State University, Portland, OR, USA \\ e-mail: courtne@pdx.edu
}

